\title{
Novel Environmental Friendly Fire Retardants
}

\author{
Alica Bartošová, Maroš Soldán*, Maroš Sirotiak, Michaela Prachová \\ Faculty of Material Science and Technology in Trnava, Slovak University of Technology in \\ Bratislava, Jána Bottu 25, 91724 Trnava, Slovakia \\ E-mail*:maros.soldan@stuba.sk
}

\begin{abstract}
Nowadays flame-retardant chemicals are mandatory in many products worldwide, flame-retardant chemicals are mandatory in many products worldwide, since they protect human life and property. Over the past few decades the use of flame-retardant chemicals has increase. Flame-retardant polymeric materials have spawned huge research interest in both scientific and industrial communities due to their broad range of applications in the fields of aviation, automotive industry, construction, electronics and telecommunications. The use of conventional FRs to meet the fire safety standards is of serious importance as they ultimately yield POPs of global concern. Considering eco-friendliness and other required properties, unsatisfactory fire performance is a major obstacle. The aim of this article is to provide an overview of traditional, commonly used flame retardants, as well as an overview of new, more environmentally acceptable alternatives.
\end{abstract}

Keywords: flame retardant; nanocomposites; natural sources.

\section{Introduction}

Fire safety is one of the most important topics in every industry (from manufacturing to construction). Every combustible material is exposed to a certain risk of fire and this risk must be minimized to the minimum. This can be achieved using non-combustible or fire-resistant materials. At present, it is mandatory for many products worldwide to add flame retardant chemicals. Flame retardants prevent ignition of the product and retard the spread of fire. They can also reduce the production of smoke and toxic smoke, which can lead to death. The prevention of fire, if successful, is more effective than merely minimizing the effects of fire.

Flame retardant systems are intended to inhibit or to stop the polymer combustion process described in the previous paragraphs. In function of their nature, flame retardant systems can either act physically (by cooling, formation of a protective layer or fuel dilution) or chemically (reaction in the condensed or gas phase).

1) Physical mechanism. The endothermic decomposition of some flame retardant additives induces a temperature decrease by heat consumption. This involves some cooling of the reaction medium to below the polymer combustion temperature. When the flame retardants decompose, with the formation of inert gases $\left(\mathrm{H}_{2} \mathrm{O}\right.$, $\mathrm{CO}_{2}, \mathrm{NH}_{3}$, etc.), the combustible gas mixture is diluted, which limits the concentration of reagents and the possibility of ignition. In addition, some flame retardant additives lead to the formation of a protective solid or gaseous layer between the gaseous phase where combustion occurs and the solid phase where thermal degradation takes place. Such a protective layer limits the transfer of matter such as combustible volatile gases and oxygen. As a result, the amount of decomposition gases produced is significantly decreased.

2) Chemical mechanism. Flame retardancy through chemical modification of the fire process can occur in either the gaseous or the condensed phase. The free-radical mechanism of the combustion process can be stopped by the incorporation of flame retardant additives that preferentially release specific radicals (e.g. $\mathrm{Cl}^{\cdot}$ and $\left.\mathrm{Br}^{-}\right)$in the gas phase. These radicals can react with highly reactive species (such as $\mathrm{H}^{\cdot}$ and $\mathrm{OH}^{\cdot}$ ) to formless reactive or even inert molecules. This modification of the combustion reaction pathway leads to a marked decrease in the exothermicity of the reaction, leading to a decrease in temperature and therefore a reduction in the fuel produced. In the condensed phase, two types of chemical reactions triggered by flame retardants are possible: first, the flame retardants can accelerate the rupture of the polymer chains. In this case, the polymer drips and thus moves away from the flame action zone. Alternatively, the flame retardant can cause the formation of a carbonized (perhaps also expanded) or vitreous layer at the surface of the polymer by chemical transformation of the degrading polymer chains. This char or vitrified layer acts as a physical insulating layer between the gas phase and the condensed phase [1].

\section{Traditional fire retardants}


The prime function of fire retardants is to either impact fire ignition or to reduce fire retardants is to either impact fire ignition or to reduce the rate of spreading of a flame. Owing to the global problem of fire emergencies, the global market for fire retardants has escalated to a multi-billion-dollar market. Flame retardancy is an active research subject developing versatile and efficient strategies to endow polymers with good fire resistant properties. The flame retardancy of any polymer is generally measured in terms of its material flammability, or the ability of a material to ignite and/or sustain combustion.

Speaking about toxicity, the public concern and the (increasingly) prohibitive legislation with regard to the persistent, bioaccumulative and toxic nature of several halogen-based compounds have divided flame retardants into two categories: halogenated and nonhalogenated. The class of halogen-free compounds is further disintegrated into smaller categories; the most frequently referred in literature are phosphorus-based flame retardants, nitrogen compounds, intumescent systems, mineral flame retardants (metal hydroxides and born compounds), silicon-based additives, and nanoparticles. Even though intumescent systems usually contain phosphorous and nitrogen substances, they perform differently than their individual counterparts; therefore, they comprise a separate class of flame retardants [2].

\subsection{Halogenated fire retardants}

The addition of halogenated FR particles is a conventional method for engineering flame retardant polymeric materials and composites. Because of their efficacy in quenching heat in either gas-phase or condensed-phase reactions, halogenated FR agents have been developed for use either as additives or copolymerized onto the polymer chains. Most of the halogenated FR agents are based on bromine or chlorine compounds, which can produce hydrogen halides that react with either hydrogen or hydroxyl radicals during combustion. Therefore, the rates of chain reactions can be reduced because the hydrogen halides inhibit exothermic oxidation reactions. [3]. Halogenated flame retardants work to remove the high-energy hydrogen $(\mathrm{H})$ and hydroxide $(\mathrm{OH})$ bond, which breaks loose during the combustion process through union with the retardant, as depicted in the following equations:

$$
\begin{aligned}
& \mathrm{H}^{\cdot}+\mathrm{O}_{2} \rightarrow \mathrm{OH}^{\cdot}+\mathrm{O} \\
& \mathrm{O}+\mathrm{H}_{2} \rightarrow \mathrm{OH}^{\cdot}+\mathrm{H}
\end{aligned}
$$

At the beginning of the process, the halogen will decompose

$$
\mathrm{RX} \rightarrow \mathrm{R} \cdot+\mathrm{X} \cdot
$$

where $\mathrm{X}$ is either chlorine or bromine. Then the halogen root will react to become hydrogen halide:

$$
\mathrm{X} \cdot+\mathrm{RH} \rightarrow \mathrm{R} \cdot+\mathrm{HX}
$$

The hydrogen halide formed interferes with the radical chain mechanism

$$
\begin{aligned}
& \mathrm{HX}+\mathrm{H} \cdot \rightarrow \mathrm{H}_{2}+\mathrm{X} \cdot \\
& \mathrm{HX}+\mathrm{OH} \rightarrow \mathrm{H}_{2} \mathrm{O}+\mathrm{X}
\end{aligned}
$$

The flame is poisoned by the freeing of halogen roots during combustion [4].

Typical representatives of the halogenated flame retardants are chlorine-, bromine-, fluorine-, and iodinecontaining compounds, with the first two comprising in tonnage terms the most widely used members of this group. This is due to the fact that efficiency and stability, depending on the kind of the halogen, are the two primary factors that determine the type of the compound that can be used as flame retardant. Fluorine- and iodine-based compounds are not employed owing to their inability to interfere with the polymer combustion process. Fluorinated organics are more stable than most commercial polymers and do not release fluorine radicals or hydrogen fluoride at the temperature range, where the decomposition of the polymers takes place [2].

\subsection{Phosphorous-based fire retardants}

The phosphorus-containing substances comprise, value-wise, one of the most commonly used groups and probably the fastest growing segment of flame retardants due to their versatility and the increasing environmental awareness of the problems arising from the use of halogen-based additives. Phosphorus-based chemicals are employed for enhancing the fire performance of thermoplastics, thermosets, textiles, paper, coatings, and mastics. Phosphorous compounds can function through various mechanisms, in the condensed polymer or in the gas phase, 
and possibly in both phases concurrently. In general, the chemical reactions involved in the decomposition of the matrix are redirected in favor of those yielding carbon rather than $\mathrm{CO}$ or $\mathrm{CO}_{2}$.

The charring mode of action of phosphorus can be exploited even in the case of poor char forming polymers, such as polyolefins and styrenics, through the introduction of a char forming additive. The acids evolved during the degradation of phosphorous-based chemicals can also form a thin glassy or liquid protective coating on the condensed phase, lowering thus the oxygen diffusion, as well as the heat and mass transfer between the gas and the condensed phase. This glassy residue may also coat the char making it more strong and cohesive, thus delaying its collapse. Even if the char does undergo oxidation (usually by smoldering), the presence of a phosphorus compound tends to inhibit complete oxidation of the carbon to carbon dioxide, thus decreasing the exothermic heat of combustion (heat sink). Some key reactions, of the hundreds possible, that have been proposed as the ones governing the gas phase action of phosphorous-based additives are the following: [2].

$$
\begin{aligned}
& \mathrm{PO}+\mathrm{H}^{\cdot} \rightarrow \mathrm{HPO} \\
& \mathrm{PO} \cdot+\mathrm{OH} \cdot \rightarrow \mathrm{HPO}_{2} \\
& \mathrm{HPO}+\mathrm{H}^{\cdot} \rightarrow \mathrm{H}_{2}+\mathrm{PO} \cdot \\
& \mathrm{OH}^{\cdot}+\mathrm{H}_{2}+\mathrm{PO} \cdot \mathrm{r} \rightarrow \mathrm{H}_{2} \mathrm{O}+\mathrm{HPO} \\
& \mathrm{HPO}_{2}+\mathrm{H}^{\cdot} \rightarrow \mathrm{H}_{2} \mathrm{O}+\mathrm{PO} \\
& \mathrm{HPO}_{2} \cdot+\mathrm{H}^{\cdot} \rightarrow \mathrm{H}_{2}+\mathrm{PO}_{2} \\
& \mathrm{HPO}_{2}+\mathrm{OH}^{\cdot} \rightarrow \mathrm{H}_{2} \mathrm{O}+\mathrm{PO}_{2}
\end{aligned}
$$

In spite of the fact that there is a scarcity of detailed investigations in the flame zone identifying the intermediate products and monitoring the concentrations of the different species generated, the main principle seems to have been understood: it is believed that the PO radical plays the major role. However, the vapourphase action of phosphorous compounds does not necessarily have to involve flame chemistry. Phosphorousbased flame retardants can either be inorganic or organic. The most commonly used inorganic compounds are red phosphorous and ammonium polyphosphate [2].

\subsection{Nitrogen-based fire retardants}

Nitrogen-containing flame retardant is a new and efficient halogen-free environmentally friendly flame retardants. There are no dioxin and halogen acids by-product and low evolution of smoke during combustion. It has better stability than those traditional flame retardants with the high bond energy between nitrogen atoms. During the thermal degradation process, it could crosslink into the char residual, and produce hardly poisonous substance [5].

Melamine is a thermally stable crystalline product characterized by a melting point as high as $345^{\circ} \mathrm{C}$ that contains $67 \mathrm{wt} \%$ nitrogen atoms. Melamine sublimates at about $350^{\circ} \mathrm{C}$. Upon sublimation, a significant amount of energy is absorbed, decreasing the temperature. At high temperature, melamine decomposes with the elimination of ammonia, which dilutes oxygen and combustible gases and leads to the formation of thermally stable condensates, known as melam, melem and melon. These reactions compete with melamine volatilization and are more pronounced if melamine volatilization is impeded, e.g. by the formation of a protective layer. The formation of melam, melem and melon generates residues in the condensed phase and results in endothermal processes, also effective for flame retardancy [6].

\subsection{Intumescent systems}

Intumescent flame retardants (IFRs) have been considered to be one of the most promising eco-friendly flame retardants because of their advantages of relatively high efficiency, low smoke, and low toxicity. Typical IFRs are comprised of three major components: an acid source, a carbonizing source, and a foaming or blowing source. For IFRs, they should decompose at a temperature lower than the thermal degradation temperature of the polymer matrix. The acid source can be one of phosphoric acid, sulphuric acid, boric acid, and halides, as well as their derivatives; the carbonizing source mainly includes pentaerythritol (monomer, dimer, and trimer), sorbitol, mannitol, dextrins, starch, phenol-formaldehyde resins, and char-forming polymers like PA-6, PU, and PC. The blowing source (agent) is primarily nitrogen-containing compounds, such as urea, urea-formaldehyde resin, melamine, dicyandiamide, and polyamides. At an elevated temperature, the acid source decomposes and generates inorganic strong acid, which can promote the dehydration of the carbonizing agent to produce the carbonaceous layer. And the quality of the carbon layer is dependent on the number of carbon atoms, while the reactive hydroxyl groups $(\mathrm{OH})$ determine the rate of dehydration reaction. Meanwhile, the blowing agent degrades and releases inflammable gases, which can expand the carbonaceous layer and make it form a swollen multicellular layer. Thus the blowing agent should decompose during the dehydration reaction of the carbonizing source in order to promote the expansion of the carbonaceous layer. Physical and chemical interactions of the 
three components lead to synergistic effect resulting in an enhanced char formation, thus leading to improved flame retardancy $[7,8]$.

\subsection{Mineral flame retardants}

Inorganic fillers are well known flame retardants and smoke suppressants for polymers in. The main advantage of inorganic fillers is that they are nontoxic. However, the flame retardant efficiency of inorganic fillers is so low that that a very high loading of filler is required to be incorporated in order to achieve an adequate flame retardancy. For example aluminium trihydrate is most commonly used inorganic flame retardant which is incorporated in amounts up to $300 \%$ of the resin level. These extremely high loadings pose processing problem and deteriorate the mechanical properties of a composite substantially.

The use of a combinations of aluminum trihydrate with ammonium polyphosphate or red phosphorus significantly reduces the loading $(\sim 50 \%)$ to achieve an optimum flame retardancy. Red phopshorus, an allotropic form of phopshorus, is not spontaneously flammable and is thermally stable up to $450{ }^{\circ} \mathrm{C}$. A silane treated grade of aluminum trihydrate (more expensive) is also available which is easier to incorporate the thermoset system [9].

The minerals most commonly used as flame retardants are metal hydroxides, borates, and hydroxycarbonates. Borates, precisely zinc borates, comprise another group of inorganic additives that have been found to impart to polymeric materials enhanced performance during combustion. They are usually depicted as a combination of oxides and water, $(\mathrm{x}) \mathrm{ZnO}$. $(\mathrm{y}) \mathrm{B}_{2} \mathrm{O}_{3} \cdot(\mathrm{z}) \mathrm{H}_{2} \mathrm{O}$, and there are at least 25 distinct members of this family, but only two of them possess characteristics (flame retarding efficiency and good thermal stability) allowing their use as flame retardants: $4 \mathrm{ZnO} .6 \mathrm{~B}_{2} \mathrm{O}_{3} .7 \mathrm{H}_{2} \mathrm{O}$ and $2 \mathrm{ZnO} .2 \mathrm{~B}_{2} \mathrm{O}_{3} .3 \mathrm{H}_{2} \mathrm{O}$. Zinc borate belonging to the second category is the most used. It works predominantly in the condensed phase promoting the formation of char, but it also performs as smoke and afterglow suppressant [2].

\subsection{Silicon-based flame retardants}

Silicon-based flame retardants are silicones, silicas, organosilicates, silsesquixanes, and silicates. Silicone flame retardants are considered to be "eco-friendly" additives because they hardly lead to harmful impacts on the environment. Typical silicones are polydimethysiloxane polymers that have excellent thermal stability, high heat release, and very low release of toxic gases like CO during thermal degradation. Their superior flame retardancy is primarily attributed to the excellent dispersion in polymer matrices and migration toward the material surface during combustion followed by the formation of a highly flame retardant char layer. Silica or silicon dioxide $\left(\mathrm{SiO}_{2}\right)$ also have various types, such as silica gel, fumed silica, and fused silica. Its effectiveness mainly depends on several factors, such as pore size, particle size, surface silanol concentration, surface area, density, and viscosity. The silica flame retardants show a significantly reduced heat release rate and mass loss rate due to the physical action of the silica in the condensed phase [7].

\subsection{Nanoparticles}

Polymer nanocomposites have recently attracted extensive attention in materials science because they often exhibit properties quite different from those of their counterpart polymer microcomposites whose matrices contain the same inorganic components. The surface areas of nanofillers are drastically increased so that polymer nanocomposites show macro/micro/nanointerfaces. Adding (CNTs) can improve not only the mechanical properties but also the functionalities such as electrical, thermal, and flammable properties of composites. Nanofiller-based flame retardants show high flame-retardant efficiencies. Adding only a small amount (i.e., $<5$ $\%$ ) of nanofiller can reduce the peak heat release rates (PHRRs) of polymers and thus reduce the speed at which flames spread throughout them. Further, the small amount of nanofiller does not reduce polymer processability and can improve the mechanical properties of polymers. The nanofillers should be combined with other conventional flame retardants to give a better balance of flammability/mechanical properties [10].

\section{Environmental friendly fire retardants}

For fire retardants to be environmentally friendly, they should not be persistent, bioaccumulative or toxic to humans, other animals and the ecosystem in general. Persistency does not necessarily mean that the chemical is detrimental to human health or that it is toxic to the environment. The important requirement is that the chemical must readily break down in the environment. Environmental transformations of persistent organic pollutants can be subdivided into three processes: biotransformation, abiotic oxidation and hydrolysis, and photolysis. The relative importance of these processes depends on the rates at which they occur under natural environmental conditions. These rates are, in turn, dependent on the chemical structure and properties of the substance and its distribution in the various compartments of the environment [6]. 
One of the most effective methods of protecting wood from fire is the use of fire retardant intumescent coatings. However, the existing fire retardants present some disadvantages related to efficiency and environmental impact. Therefore, research is focused on the chemical modification of natural polymers and wood, and on the development of more efficient intumescent systems and fire protectors.

Flame retardant property is important especially for polymer products, which various flame retardant materials can be applied for suitable products. As well as, using of biodegradable polymers to replace or partially replace conventional polymers has been considered as an effective way to minimize the impact of plastic products disposal on the environment. Poly (lactic acid) (PLA), is the most common conventional polymer substitute due to its good mechanical properties and non-microbial degradation process. Neat PLA homopolymer ignites easily, supports continued combustion, and does not form a char. Hence before the polymer can be widely applicable as a substitute for non-biodegradable materials, its flame retardant properties must be improved [11].

Biobased polymers have been defined as human-made or human-processed macromolecules derived from biological resources for plastic or fibre applications. Various types of partially or completely biobased thermosetting and thermoplastic polymers are already commercially available. Among the thermoplastics mainly polyesters and polysaccharide derivatives can be found; these include biobased PET, PLA, biobased PE, starch blends, polyhydroxy alkanoate (PHA), biobased polyamide, cellulose acetate, cellulose acetate propionate, cellulose acetate butyrate and poly $\varepsilon$-caprolactone (PCL). PCL is compatible with many polymers and thus is used in many formulations as a compatibilizer, or it provides water resistance in starch-based formulations.

\subsection{Retardants from biomass}

Fire retardant additives have high-value applications for which some biomass constituents are predisposed. In nature, certain lignocellulosic plants have developed defense behaviors against fire aggression. This is the case of cork oak that is more fire-resistant than other trees owing to the slow combustion of cork, containing mainly suberin (waxy substance composed of polyaliphatic and polyaromatic domains interlinked by glycerol), cellulose and lignin. These bio-based compounds owe their advantages as flame retardant additives to their molecular composition and structure providing them inherent ability to produce thermally stable charred residues when exposed to fire.

The char formation during the burning of wood is well known. When exposed to heat, the temperature of the wood surface increases, the water adsorbed in the wood starts to be released and the decomposition of the wood constituents, i.e. lignin, cellulose and hemicelluloses, begins around $160-180{ }^{\circ} \mathrm{C}$. At $350-360{ }^{\circ} \mathrm{C}$, ignition of the wood can occur in the presence of a spark, and the accelerated degradation leads to the formation of a charred layer which acts as an insulator for the underlying wood [12].

\subsubsection{Cellulose}

Cellulose is a long chain linear homopolymer comprising anhydro- $\beta$-d-glucopyranose units linked by $(1 \rightarrow 4)$ glycosidic bonds. It is the most abundant raw organic material in the nature, presents interesting char forming properties. The improvement of fire performances lies on the capacity of treated cellulose to form carbonaceous char along the combustion step. The first step, occurring below $280{ }^{\circ} \mathrm{C}$ and leading to the formation of anhydrocellulose, is enhanced for low temperatures and a slow heating rate. At higher temperatures, a competitive, more endothermic unzipping reaction is initiated for the remaining cellulose. This unzipping reaction leads to the formation of tar, while anhydrocellulose undergoes an exothermic decomposition resulting in the formation of char and gas (third step) [12].

Dorez et al. [13] studied the pyrolysis and combustion of natural fibers and they found out that a low content of lignin with a high content of cellulose lead to charring by affecting the degradation pathway of cellulose. Fox et al. [14] reported on the performances of cellulose materials used in its nanofibrillated form as a carbon source in intumescent flame retardant system for PLA [15].

The presence of other components during the pyrolysis of cellulose also influences its degradation pathway. Some inorganic salts, naturally present in the biomass, such as mineral compounds in the ash, promote the formation of condensed structures through the catalysis of the dehydration reactions of cellulose upstream from the depolymerization reactions, enhancing the yield of solid products.

Several studies have demonstrated a significant catalytic effect inducing a decrease in the first degradation temperature and an increase in the amount of char obtained by combining cellulose and some inorganic salts such as $\mathrm{CuSO}_{4}, \mathrm{NaOH}, \mathrm{Na}_{2} \mathrm{CO}_{3}, \mathrm{NiCl}_{2}, \mathrm{NaCl}, \mathrm{MgCl}_{2}, \mathrm{ZnCl}_{2}, \mathrm{~K}_{2} \mathrm{CO}_{3}$, and $\mathrm{KCl}$ [12].

\subsubsection{Hemicellulose}

Hemicellulose is the second most abundant polysaccharide after cellulose in plant cell walls, accounting for $15-30 \%$ of lignocellulosic biomass by weight. In general, the amounts of hemicellulose in wood and woody 
biomass are greater than those in herbaceous and agricultural biomass [16]. Due to the low molecular weight of hemicellulose, its thermal decomposition is rapid and starts around $180{ }^{\circ} \mathrm{C}$, with the maximum weight loss rate between 250 and $350{ }^{\circ} \mathrm{C}$. During the thermal degradation of hemicellulose, the release of adsorbed water is accompanied by the cleavage of weak side branches.

The maximum weight loss is obtained by dehydration, decarboxyl- ation, and decarbonylation reactions, which lead to the release of volatile gases, such as $\mathrm{CO}_{2}, \mathrm{CO}, \mathrm{H}_{2} \mathrm{O}$, acetic acid, and furfural. During the main thermal degradation step, some random repolymerization between the radicals produced occurs and results in the formation of a large amount of char. The better char-forming ability of hemicellulose, compared to cellulose, could be explained by the formation of unstable radicals, after the cleavage of glycosidic linkages, due to the absence of a sixth carbon and oxygen at the $\mathrm{C} 4$, inhibiting the formation of a stabilized anhydride. This instability leads to subsequent reactions, such as transglucosylation and dehydration, to form char [12].

\subsubsection{Starch}

Flame retardant property is important especially for polymer products, which various flame retardant materials can be applied for suitable products. Whatever the nature of native starch, its thermal degradation occurs in three stages. The first step corresponds to a physical dehydration related to the release of absorbed water. The second stage, which starts at around $300{ }^{\circ} \mathrm{C}$, concerns chemical dehydration and thermal decomposition, with thermal condensation between hydroxyl groups, leading to the formation of ether segments and water liberation. Dehydration of neighboring hydroxyl groups in the glucose ring also occurs, resulting in the formation of $\mathrm{C}=\mathrm{C}$ bonds or ring scission. Aromatic rings, such as interconnected benzene and furan structures are formed at higher temperature. The last stage takes place above $500{ }^{\circ} \mathrm{C}$, with carbonization reactions and the formation of large conjugated aromatic structures. Starch has been mainly used as a carbon source in intumescent flame retardant systems [12].

\subsection{Nano-based fire retardants}

Nanotechnology is the science, engineering, and technology conducted at the nanoscale, (which is about 1 to $100 \mathrm{~nm}$ ) that enables the manipulation of materials to fit existing needs. Nanotechnology is being used in brick, mortar and other clay related products to achieve a low thermal conductivity, high gas barrier properties and a delayed char formation. Using this nano-paper in clay products the decrease in thermal conductivity and increase in delayed char formation will achieve an overall increase in fire retardant abilities. Clay nanocomposites, which are also made with clay nanotechnology, are prepared by simple filtration and used mainly in brick and mortars. The clay nanocomposites have superior fire protection, a higher resistance to flammability and higher exposure to heat flux and also have thermal and thermo-oxidative stability in nitrogen and oxidative atmospheres. The clay nanocomposites can be used in interior and exterior applications [17, 18].

\subsubsection{Hydrogels}

Hydrogels are gelatinous amalgams of cross-linked polymers that can absorb and hold large quantities of water. Hydrogels are used as a secondary layer and are laminated to fabrics to create a fire-resistant material. Hydrogels contain $90 \%$ water, which has a larger heat capacity and allows for a larger amount of energy to be absorbed from a fire. This is done by the hydrogel heating from the fire and evaporating the water, therefore, the clothing and other apparel provides a prolong exposure in fires for fire fighters and other fire personal. The downside to hydrogels are they can be less expensive but also less effective while also decomposing faster when exposed to higher temperatures [18].

\subsubsection{Carbon-based nanomaterials}

Carbon-based materials comprise an attractive and fascinating family, including the naturally abundant and cost-effective graphite, and also the most expensive diamond. Up to now, carbon-based materials include carbon black, graphite, graphene, CNT (carbon nanotubes), and fullerene. These materials are not isolated from each other, but in close relationship. For example, graphene, the latest member in this family, is an atomically thick, two-dimensional (2-D) sheet, regarded as the building block of all other graphitic carbon allotropes with different dimensionality : 3-D graphite is made of graphene sheets stacked on top of each other with a spacing distance of 3.37 Aं; 1-D CNT may be made by rolling graphene sheets; 0-D fullerenes (buckyballs) may be envisioned to be made by wrapping a part of graphene sheet (0-D refers to the lack of translational symmetry in any dimension). Due to their extraordinary intrinsic properties and ability to be dispersed in various polymer matrices, carbonbased materials have been widely used in fabricating polymer composites/nanocomposites in order to improve the mechanical, thermal, gas barrier, conductivity, and flame retardant performances [19]. 
CNTs are a promising alternative to the use of conventional flame retardants. It could be adding a small amount (usually $<5 \mathrm{wt} \%$ ) of carbon nanotubes for reduce the fire hazards for a wide range of polymers such as PP, PE, EVA, PS, PLA, PMMA, PA-6, PC, PEEK, polybenzoxazine and epoxy resin.

Graphene, as a new member of carbon allotropes discovered by the exfoliation of graphite in 2004 [20], has been arousing tremendous attention and research interest in the scientific community. With the unique structure of a 2-D monolayer of $\mathrm{sp}^{2}$ hybridized carbon atoms, graphene has shown exceptional physical properties what make graphene one promising nanofiller to be employed in nanocomposites for many multi-functional applications. Graphene with high purity is quite stable against combustion when exposed to a natural gas flame for a few seconds. The burned part turns red hot but does not spread, and is quenched after the removal of the flame, clearly indicating the high intrinsic flame resistance of graphene. Besides, graphene phosphonic acid has been prepared by a simple method of ball-milling graphite with red phosphorus that is demonstrated as an effective flame retardant [19]. The flame retardant action of graphene is mainly focused on its influence on the structure and yield of the char formed.

Graphite is a layered mineral made up of stacked graphene sheets where carbon atoms within the layered nano-sheets form hexagonal cells through covalent bonds, with successive carbon layers connected by Van der Waals forces. However, pristine graphite is rarely used in flame retardant polymers since the carbon flakes in natural graphite stack so compactly that the penetration of the resin matrix into the slots of the graphite sheets is very difficult. Instead, expandable graphite (EG) prepared from natural graphite by chemical treatment has been widely used as flame retardant for a wide range of polymers due to its easy exfoliation in a polymer matrix. The utilization of EG as a flame retardant benefits from its layered structure ('physical' barrier action) and intumescent/blowing effect ('chemical' action) in the condensed phase. When exposed to heat, EG, expands hundred times its initial volume and generates a "worm-like" structure layer on the surface of the materials. Such a layer of graphite can prevent heat and oxygen permeating into the polymer matrix, thus improving the fire resistance [19].

\section{Results}

Flame retardants (FRs) inhibit ignition, slow the spread of fire and protect critical infrastructure during a fire event. They also can reduce smoke and toxic fume production that can lead to death in fire. Over $60 \%$ of all FR plastic formulations are still based on halogen-containing fire retardants additives, even though these materials have some severe, undesirable side effects, such as persistence in the environment, bio-accumulation in animal and human tissues, and cytotoxicity. The development of alternative active components of flame retardants is currently attracting huge interest of many researchers from around the world. The development and production of environmentally friendly flame retardants is relatively demanding. Attention is focused mainly on the use of nanocomposites. One of the most important ways to achieve sustainable materials development, as well as the search for new types of retarders on a natural basis.

\section{Acknowledgment}

This paper was supported by the project Young researcher AFTOPSD Assessment of the factors of technological operations in the production of fire-fighting equipment door

\section{References}

[1] Laoutid, F. at al. New prospects in flame retardant polymer materials: From fundamentals to nanocomposites. Materials Science and Engineering R 632009 p.100-125

[2] Papaspyrides, C., Kiliaris, P., Polymer Green Flame Retardants. Elsevier 2014 ISBN: 978-0-444-53808-6

[3] Vikas, M., Thermally Stable and Flame Retardant Polymer Nanocomposites. Cambridge University Press 2011 ISBN 978-0-521-19075-6

[4] Njuguna, J., Lightweight Composite Structures in Transport - Design, Manufacturing, Analysis and Performance. Elsevier 2016 ISBN 978-1-78242-325-6

[5] Qian, X., Liu, H., Advanced Textile Materials, Part 1. Trans Tech Publications Ltd. 2011 ISBN 978-303785-241-

[6] Blackburn, R. S. Sustainable Textiles - Life Cycle and Environmental Impact. Woodhead Publishing. 2009 ISBN 978-1-84569-453-1 
[7] Fan, M., Fu, F., Advanced High Strength Natural Fibre Composites in Construction. Elsevier 2017 ISBN 978-0-08-100411-1

[8] Ratna, D., Epoxy Composites - Impact Resistance and Flame Retardancy. Smithers Rapra Technology 2007 ISBN 978-1-84735-065-7

[9] Ratna, D., Handbook of Thermoset Resins. Smithers Rapra Technology 2009 ISBN 978-1-84735-410-5

[10] Arao, Y., Flame Retardants. Springer 2015

[11] Guo, Z. et al. Incorporation of cellulose with adsorbed phosphates into poly (lactic acid) for enhanced mechanical and flame retardant properties. Polymer Degradation and Stability 2017. Volume 144, p. 24-32

[12] Costes, L. et al. Bio-based flame retardants: When nature meets fire protection. Materials Science and Engineering R 2017, p.1-25

[13] Dorez, G. et al. Effect of cellulose, hemicellulose and lignin contents on pyrolysis and combustion of natural fibers. Journal of Analytical and Applied Pyrolysis 2014. Volume 17, p.323-331

[14] Fox, D.M. et al. Flame retarded poly (lactic acid) using POSS-modified cellulose. 1. Thermal and combustion properties of intumescing composite. Polymer Degradation and Stability 2013. Volume 98, p. 590-596

[15] Costes, L. et al. Cellulose/phosphorus combinations for sustainable fire retarded polylactide. European Polymer Journal 2016. Volume 74, p.218-228

[16] Luo, Y. et al. The production of furfural directly from hemicellulose in lignocellulosic biomass: A review. Catalysis Today 2019. Volume 319, p.14-24

[17] Wang, D-Y. Novel Fire Retardant Polymers and Composite Materials. Elsevier 2017 ISBN 978-0-08100977-2

[18] Olawoyin, R. Nanotechnology: The future of fire safety. Safety Science 2018. Volume 110, p.214-221

[19] Wang, X. et al. Carbon-family materials for flame retardant polymeric materials. Progress in Polymer Science 2017, Volume 69, p. 22-46

[20] Novoselov, K.S at al. Electric field effect in atomically thin carbon films. Science 2004, p. 666-669 